\title{
A Study Protocol to Assess Viral Load Suppression Rate among Adolescents Leaving With HIV
}

\author{
Article by Tibananuka Evelyne \\ Masters of Public Health, Texila American University, Uganda \\ E-mail:evakiiki@gmail.com
}

\begin{abstract}
\section{Introduction}

Assessing viral load suppression among adolescents leaving with HIV in Uganda. The term "viral load" refers to the number of copies of HIV per $m L$ of blood. In other words, it's the amount of virus in the blood. Provision of Antiretroviral drugs (ARV') to people leaving with HIV aims mainly at achieving viral suppression. ART are medications that treat HIV. The drugs do not kill or cure the virus. However, when taken in combination they can prevent the growth of the virus. When the virus is slowed down, it is called viral suppression and this also slows down HIV disease.

According to United Nation's Programme on HIV/AIDS in 2013, 90-90-90 is a set of goals. The idea is that by 2020, 90\% of people who are HIV infected will be diagnosed, $90 \%$ of people who are diagnosed will be on antiretroviral treatment and $90 \%$ of those who receive antiretroviral will be virally suppressed.

Viral suppression is defined as, literally, suppressing or reducing the function and replication of a virus. Even if no virus is present in the blood, HIV infected cells may remain in the body. Therefore, there is the possibility that the virus could begin to replicate (copy itself) again if antiretroviral therapy were to be stopped. Furthermore, having an "undetectable" viral load simply means that there are too few copies of the virus to be detected by current tests As such, "undetectable" is a moving target. Viral suppression is achieved by 12 weeks of initiation on antiretroviral (ARV) therapy in patients who have 95\% adherence.
\end{abstract}

Unsuppressed Viral load. Using WHO criteria, virological treatment failure is defined as having detectable VL after 6 months of adherence to ART beyond 1000 copies $/ \mathrm{ml}$. This is also called unsuppressed viral load.

In 2016, HIV prevalence in Uganda currently stands at 7.3 percent up from the 6.2 percent when Uganda had made strides in HIV prevention.

According to the Ministry of health, Uganda registers 120,000 new HIV infections every year, more than 1.5 million Ugandans are estimated to be living with HIV and AIDS.

Out of those infected so far, 84 percent transmission of HIV is through unprotected sex, 14 percent is mother-to-child-transmission (MTC), while 2 percent is accidental or through other modes of transmission.

There remain uncertainties as to whether all adolescent needs on ART have HIV viral suppression after six months of treatment. This project will assess the accessibility, utilization and compliance of adolescents (aged 10-19 years) to ARVs from selected HIV clinics in Uganda, their viral load monitoring and suppression. The study results shall inform advocacy and programming of adolescent friendly services in HIV clinics by humanitarian partners.

\section{Problem statement and justification}

Stigma and disclosure remain the biggest challenge among adolescents in Uganda. According to Christine Aruo, the Adolescent and Child Counselor at The Aids Support Organization (TASO) disclosed that the high viral load is arising greatly due to the failure by parents to disclose to their children about their HIV status. "One of the things we have 
observed right now as we are doing viral load is that in many of our adolescents, and our children, the viral load is high, it has not been suppressed, and as a team we've been wondering why", Aruo noted.

"It is because the parents or care takers have not opened up to their children about their HIV status hence many children living with HIV are not adequately taking their antiretroviral drugs (ARV's) as expected.”, she said.

Charles Opolot the Public Relations Advocacy and Communication's Officer Baylor Uganda said that 90-90-90 strategy is achievable only if the media strengthened advocacy in ensuring that the communities changed their behavior towards HIV prevention.

When taking any drug ARVs inclusive, one should adhere to the right dose, route and time. This means that if we are to achieve the 90-90-90 UNAIDS strategy by 2020, all people on ARVs including adolescents have to take their medications in the right dose, at the right time and through the right route as per prescription. Any missed or delayed dose gives HIV a chance to multiply and replicated and this may eventually lead to unsuppressed viral load.

According to the The AIDS Support Organisation (TASO) Masindi is one of the leading HIV/AIDS service providers in Mid-Western Uganda. TASO Masindi is also one of the eleven TASO Centres that are operating in Uganda and has been in existence since 2005. The centre serves more than three hundred (300) adolescents and half of them have un suppressed viral load. It also supports Masindi Hospital to Provide Adolescent Friendly Services. The centre is challenged with high viral load among adolescents accessing HIV/AIDS care. Nearly a half of our adolescents are not suppressing their viral load greatly due to poor adherence among this key group propagated by fear to disclose to their partners, parents teachers especially those in boarding schools who even end up missing their appointments sometimes, among others. This is a contributing factor to new HIV infection among this group. In Uganda, it was noted that 350 adolescents acquire HIV infection every week (MoH, 2015).

\section{Methodology}

The search criteria will include; WebMD, Medline plus, Nam iadsmap, Cochrane libraries, PubMed, and others. Literature will be organized and referenced by research software like Mendeley. A cross-sectional study using qualitative and quantitative approaches will be conducted. Results will be analyzed using United Nation's AIDS Programme and WHO recommended expand-net framework.

Ethics: Participation in an assessment will be voluntary. Parental consent for minors will be obtained before participating in the study.

Approval from Uganda Ministry of Health ethical committee shall be sought.

Foot note: The cross-section, Complex. Humanitarian, Adolescent, HIV in Uganda.

\section{Data collection}

This will involve a summary of existing data, previous research reports, books, journals, government and partner NGO statistics and online databases.

\section{Study selection}

\section{Our study population}

The participants in this study will be composed of both in and out of school adolescents aged (10-19 years). Our focus of the investigation will include; adolescents themselves, the Ministry of Health (MOH) staffs, None Governmental Organizations staff in Country response clusters, parents of these adolescents and their guardians, their teachers and religious as well as cultural leaders among others.

\section{Recommendation}

To have trained adolescent counsellors and all facilities with ART services to start up HIV adolescent friendly clinics which will always; Provide improved psychosocial support to 
children and adolescents, adolescent friendly individual and group counselling sessions, conducts home visits to support children and adolescents on adherence, links needy children to support agencies and provides child rights education to Orphans and Other vulnerable (OVC) households ,delivers ARVs to their homes for those that miss appointment. Have child/adolescent focal persons at all levels, the focal person will be entirely responsible for children and adolescents' activities and these are her output indicators for her daily performance and will help to achieve the 90/90/90.

\section{References}

[1]. https://aidsinfo.nih.gov/guidelines/html/1/adult-and-adolescent-arvguidelines/15/virologic-failure

[2]. The United Nations Joint Programme on HIV/AIDS (UNAIDS)

[3]. https://za.usembassy.gov/wp-content/uploads/sites/19/2016/06/Innovations-in-

Identifying-and-Managing-Unsppressed-Viral-Load-Strategies-and-Processes_-Chazanga-

Tembo.pdf

http://www.aidsmap.com/

https://www.verywell.com/

https://tekauganda.org/rise-in-viral-load-among-adolescents-in-soroti-worries-medics/

[4]. JAIDS Journal of Acquired Immune Deficiency Syndromes: 1 October 2007 - Volume

46 - Issue 2 - pp 187-193 doi: 10.1097/QAI.0b013e31814278c0

http://journals.lww.com/jaids/pages/default.aspx

[5]. https://tekauganda.org/rise-in-viral-load-among-adolescents-in-soroti-worries-medics/ 